\title{
The Complete Surgical Resection Without the Radiotherapy for a Recurred Anaplastic Ependymoma at the Cervicomedullary Junction
}

\author{
Chang Sun Lee, Chul Kyu Lee, Ki Hong Jo, Sang Hyun Kim \\ Department of Neurosurgery, Ajou University School of Medicine, Suwon, Korea
}

The intramedullary anaplastic ependymoma rarely occurs in the cervicomedullary junction. A 45-year-old woman had a history of right arm pain for several months. Magnetic resonance imaging (MRI) of the cervical spine demonstrated an intramedullary tumor with syrinx at the cervicomedullary junction. The patient underwent a partial resection at another institute. Neurologic deficit worsened after the first surgery. The follow up MRI showed that the enlarged enhancing tumor and syrinx still existed with the same size and configuration. Complete surgical resection was achieved in the revision surgery. Final histologic examination confirmed the diagnosis of an anaplastic ependymoma, and since complete surgical resection was achieved the patient did not receive adjuvant radiation or chemotherapy. The patient was followed-up periodically at the outpatient department, and at the 7 months follow-up the muscle tone of the right hand was normal but with mild sensory deficit, and the MRI demonstrated no evidence of recurrent disease. Intramedullary anaplastic ependymoma that occur in the cervicomedullary junction which are completely resected may be followed-up without adjuvant radiation or chemotherapy to attain good clinical outcome.

Key Words: Anaplastic $\cdot$ Ependymoma $\cdot$ Cervicomedullary junction

\section{INTRODUCTION}

Ependymomas are the most common intramedullary tumor in adults, and they account for $60 \%$ of intramedullary spinal cord tumors $^{14)}$. Intramedullary spinal cord ependymomas arises from the ependymal cells lining the central $\mathrm{canal}^{6}$. Intramedullary ependymomas are most commonly observed in the cervical and cervico-thoracic regions. Spinal cord ependymomas are usually benign and curable with complete surgical resection or in combination with radiotherapy ${ }^{2,11)}$. However the anaplastic ependymomas frequently recur and metastasize and therefore adjuvant radiotherapy should be considered even after complete surgical resection ${ }^{13)}$.

Administration of postoperative adjuvant radiotherapy may result in life-threatening or serious neurologic deficits ${ }^{8)}$, and those occurring in the cervicomedullary junction are particularly severe.

- Received: July 19, 2012 • Revised: September 21, 2012

- Accepted: September 25, 2012

Corresponding Author: Sang Hyun Kim, MD

Department of Neurosurgery, Ajou University School of Medicine, 5

Wonchan-Dong, Youngtong-Gu, Suwon 442-721, Korea

Tel: +82-31-219-5230/5232, Fax: +82-31-219-5238

Email: shkim709@ajou.ac.kr
We report herein a case of an anaplastic ependymoma located in the cervicomedullary junction, who experienced recurrent disease after initial partial resection and underwent repeat surgery to achieve complete resection, and who showed good clinical outcome without adjuvant radio- therapy.

\section{CASE REPORT}

A 45-year-old woman developed right arm pain which radiated down to all 5 finger tips for several months initially. The past medical history was unremarkable. She had visited a local hospital and the magnetic resonance imaging (MRI) demonstrated a solid intramedullary tumor with syrinx at the cervicomedullary junction which was well enhanced by gadolinium contrast media (Fig. 1). The well-enhanced tumor was located at the ventral portion of the cord and the linear syrinx was located from the cervicomedullary junction to C7. For tumor removal, a suboccipital decompression and removal of the tumor was performed at the local hospital. The pathologic finding was an ependymoma at that time. After the first surgery, she suffered from pain and tingling sensation of her right arm which gradually worsened. The follow-up MRI 6 months later after the first surgery revealed an enlarged enhancing tumor and syrinx compared with initial MRI (Fig. 2). The patient 

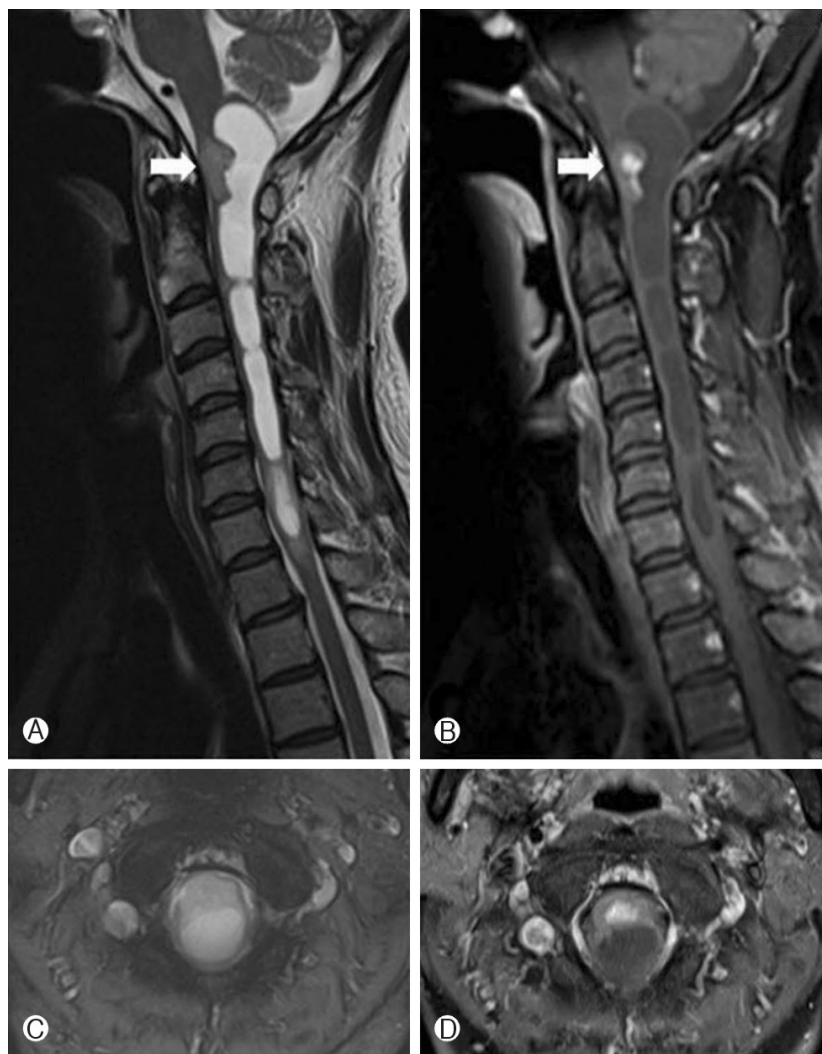

Fig 1. $A$ and $B: T 1$-weighted sagittal magnetic resonance (MR) image (A) and T2-weighted sagittal MR image (B) before the surgery show an cervicomedullary junctional mass (white arrow). (C) and (D) On the pre-operative enhanced MR image.

was referred to our institution and the initial neurologic examination demonstrated that she had grade 4 motor weakness of her right arm with tingling sensations. Revision surgery was performed. The tumor was seen to be originating from the medulla oblongata to the $\mathrm{C} 1$ spinal cord with a huge sized syrinx. The scar tissue was intermingled with neural tissue on the upper, lower and dorsal portions of the tumor. But the ventral portion of the tumor showed a clear demarcation between the tumor and normal neural tissue. Gross total resection of tumor was achieved, despite the previous surgery. The pathological diagnosis was an anaplastic ependymoma (Fig. 3) and the MIB-1 index was increased mildly. Immediate after the surgery, the patient showed mild hemipaparesis of her right side. In consideration of the detrimental effects of radiotherapy on the twice-surgery area we opted for shortterm follow-up rather than adjuvant radiotherapy. On the follow-up MRI at 3 months and 7 months after the surgery, no residual or recurrent tumor was observed (Fig. 4). The right side hemiparesis which occurred immediately after surgery improved such that normal everyday activity was almost normal 1 month after the surgery, and at post operative 8 months
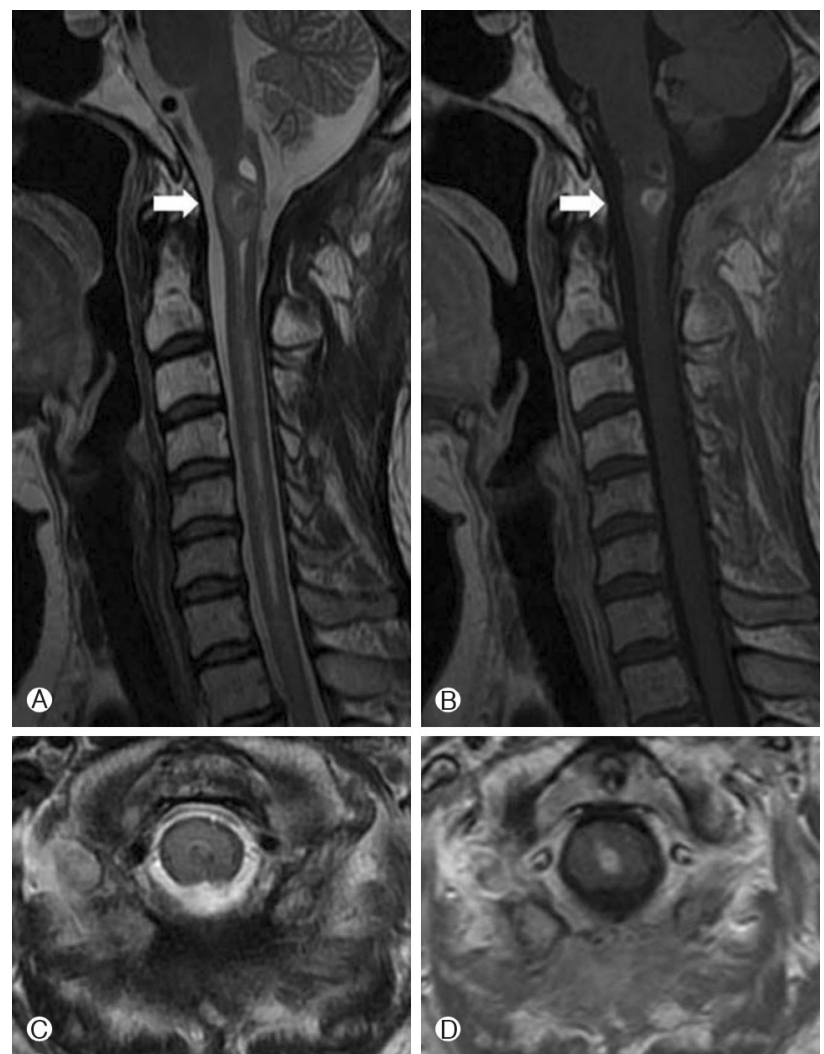

Fig. 2. T1-weighted sagittal MR image (A) and T2-weighted sagittal MR image (B) after the initial surgery show a cervicomedullary junctional mass (white arrow). C and D: Recurrent tumor on the postoperative enhanced MR image.

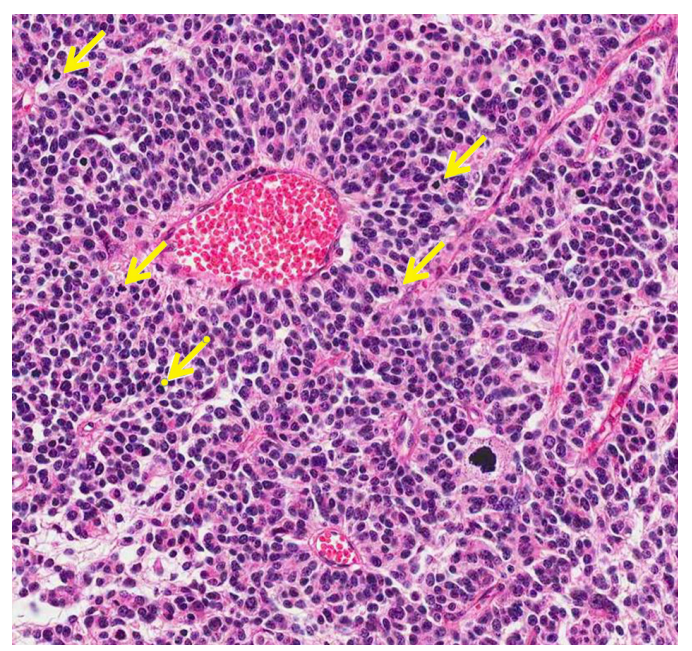

Fig. 3. Ependymoma showing area of increased cellularity, mitoses (Yellow arrow), and cellular atypia (hematoxylin and eosin stain 200).

the neurologic evaluation in the follow-up period did not reveal any new or residual deficits. 

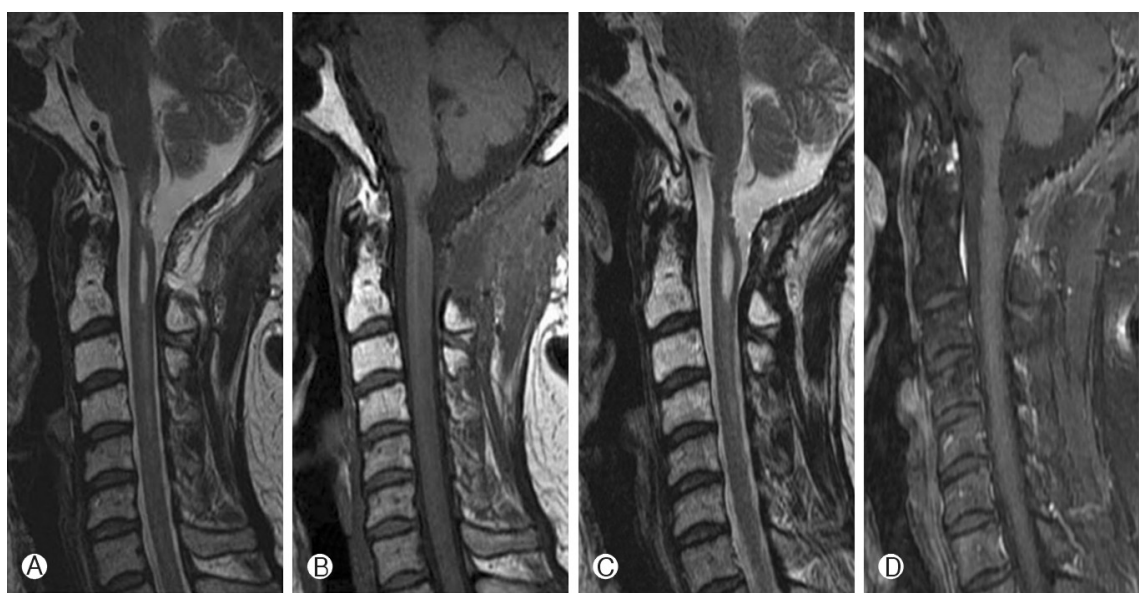

Fig. 4. T2 - (A) and T1- (B) weighted sagittal MR 3 months after the second surgery. T2( $\mathrm{C}$ and T1- (D) weighted sagittal MR 7 months after the second surgery. There was no residual or recurrent tumor with well decompression of the syrinx.

\section{DISCUSSION}

Ependymoma is the most common intraspinal tumor ${ }^{14)}$. Ependymomas arise from ependymal cells that line the ventricles and central canal of the spinal cord ${ }^{13,14}$. It comprises $15 \%$ of spinal cord tumors and up to $60 \%$ of spinal cord gliomas ${ }^{2)}$. A previous study reported the incidence as four regions were localized in the thoracic region (19\%), 7 in the lumbar region (34\%), 4 in the cervical region (19\%), 3 in the cervicothoracic region (14\%), and 3 in the conus medullaris $(14 \%)^{14)}$. In the same study, pathological examinations revealed that 7 (34\%) lumbar region cases were identified as myxopapillary ependymomas stemming from the filum terminale, and 14 (66\%) other cases were intramedullary ependymomas. There were no malignant ependymoma cases ${ }^{14)}$.

Several cases of clear cell ependymoma of the medulla oblongata has been reported and also a case of cervicomedullary junction tanycytic ependymoma ${ }^{3,8)}$. In general, ependymomas includes anaplastic (malignant) ependymomas, and myxopapillary ependymomas, cellular, papillary, clear cell, and tanycytic subtypes ${ }^{1,5,7,10,13)}$. Almost all spinal cord ependymomas are histologically benign tumors ${ }^{2,13)}$ and curable with complete surgical resection or in combination with radiotherapy ${ }^{16}$. Maximal resection of the tumor while protecting neurological function is the golden rule in surgical treatment of spinal ependymomas $^{5)}$. Also, anaplastic ependymomas are less common and has worse prognosis ${ }^{5,14,16)}$.

In our present case, tumor originated from the medulla oblongata to the $\mathrm{C} 1$ spinal cord with a huge sized syrinx. Gross total removal (GTR) of the tumor was successfully achieved in spite of the previous surgical treatment and location of cervicomedullary junction. The pathology was confirmed as anaplastic ependymoma.
Careful manipulation of the tumor results in reducing the postoperative neurologic deficit and a significant favorable clinical outcome. As anaplastic ependymomas frequently recur and is accompanied with a poor prognosis, it is important that they are totally removed grossly in order to achieve a good outcome $^{5,12)}$. In the operation field, clear demarcation was observed between the tumor and neural tissue, and therefore complete resection could be achieved. After the complete resection, we determined that conservative management without adjuvant radiotherapy would be the best therapeutic plan for the patient.

The complete resection alone in spinal cord "ependymoma" without local radiotherapy can achieve excellent tumor control and survival". Since a residual tumor or "malignant ependymoma" has a poor prognosis, postoperative radiotherapy is recommended in the past literature review. ${ }^{10,11,15)}$. The majority of reports propose that radiotherapy in those who have a "anaplastic ependymoma" should be added to the surgical resection $^{13)}$.

The ependymoma of this patient was located at the cervicomedullary junction and therefore consideration of the possibility of the radiotherapy induced central nervous system injury should be taken into account. In addition, information pertaining to the scope and effect of radiation therapy is lacking and remains under debate ${ }^{8)}$. The radiation therapy may result in the gliosis, fibrosis and hardening of ependymoma ${ }^{4}$. The radiation therapy can also predispose the patient radiation induced myelopathy and result in the severe neurologic damage ${ }^{4}$. As the mild motor weakness that was observed in the immediate post-operation period was expected to improve with rehabilitative therapy, and because of the possibility of neurologic deficit and delayed recovery, radiotherapy was not included in the postoperative treatment plans. During the follow-up period, no new neurologic symptoms appeared, the neurologic deficit improved, and the MRI demonstrated that 
there was no residual or recurrent tumor.

We agree that adjuvant radiotherapy is necessary in patient with anaplastic ependymomas. However, in the group of patients in whom radiotherapy is envisaged to consequently result in severe neurological deficits, complete resection and shortterm follow up may be a valid alternative.

\section{CONCLUSION}

Anaplastic ependymoma arising from the cervicomedullary junction is uncommon. Surgical resection should be considered as an initial treatment for residual or recurred anaplastic ependymomas, followed by postoperative radiotherapy. Adjuvant radiotherapy in patients with complete resected anaplastic ependymoma may not always be necessary. In patients who received the surgical resection at the cervicomedullary junction, complete resection alone might be a strategic recommendation.

\section{REFERENCES}

1. Amatya VJ, Takeshima Y, Kaneko M, Nakano T, Yamaguchi S, Sugiyama K, et al: Case of clear cell ependymoma of medulla oblongata: clinicopathological and immunohistochemical study with literature review. Pathol Int 53:297-302, 2003

2. Brescia MA, Di Martino G, Guillou C, Reniero F, Sacco A, Serra F: Differentiation of the geographical origin of durum wheat semolina samples on the basis of isotopic composition. Rapid Commun Mass Spectrom 16:2286-2290, 2002

3. Delaloye JF, Seraj F, Guillou L, Genton CY, Anciaux-Le Teno D, Schnyder P, et al: Granular cell tumor of the breast: a diagnostic pitfall. Breast 11:316-319, 2002

4. Nagasawa DT, Smith ZA, Cremer N, Fong C, Lu DC, Yang I: Complications associated with the treatment for spinal ependymomas. Neurosurg focus 31(4):E13, 2011

5. Eliat C, Lassel L, Guillou YM, Le Bouar G: [Intravenous beta-2adrenergic agonists for tocolytic therapy in preeclampsia: two cases of acute pulmonary edema]. Ann Fr Anesth Reanim 21: 737-740, 2002

6. Guilleret I, Yan P, Guillou L, Braunschweig R, Coindre JM, Benhattar J: The human telomerase RNA gene (hTERC) is regulated during carcinogenesis but is not dependent on DNA methylation. Carcinogenesis 23:2025-2030, 2002

7. Guyotat J, Metellus P, Giorgi R, Barrie M, Jouvet A, FevreMontange $\mathrm{M}$, et al: Infratentorial ependymomas: prognostic factors and outcome analysis in a multi-center retrospective series of 106 adult patients. Acta neurochirurgica (Wien) 151:947960, 2009

8. Stuben G,Stuscheke M, Kroll M,Havers W, Sack H: Postoperative radiotherapy of spinal and intracranial epnedymomas: analysis of prognostic factors. Radiother Oncol 45:3-10, 1977

9. Ito T, Ozaki Y, Nakagawara J, Nakamura H, Tanaka S, Nagashima K: A case of cervicomedullary junction tanycytic ependymoma associated with marked cyst formation. Brain Tumor Pathology 22:29-33, 2005

10. Kaner T, Sasani M, Oktenoglu T, Solmaz B, Sarloglu AC, Ozer AF: Clinical analysis of 21 cases of spinal cord ependymoma: positive clinical results of gross total resection. J Korean Neurosurg Soc 47:102-106, 2010

11. Lin YH, Huang CI, Wong TT, Chen MH, Shiau CY, Wang LW, et al: Treatment of spinal cord ependymomas by surgery with or without postoperative radiotherapy. J Neurooncol 71:205210, 2005

12. Nam Hoon Y, Seong Rok H, Sang Won Y, Gi Taek Y, Chang Young C, Moon Jun S, et al: Spinal intramedullary Ependymoma. Korean J Spine 2(3):199-203, 2005

13. Niazi TN, Jensen EM, Jensen RL: WHO Grade II and III supratentorial hemispheric ependymomas in adults: case series and review of treatment options. J Neurooncol 91:323-328, 2009

14. O'Leary R, Wood EJ, Guillou PJ: Pathological scarring: strategic interventions. Eur J Surg 168:523-534, 2002

15. Ohata K, Takami T, Gotou T, El-Bahy K, Morino M, Maeda M, et al: Surgical outcome of intramedullary spinal cord ependymoma. Acta Neurochir (Wien) 141:341-346; discussion 346-347, 1999

16. Slater GW, Guillouzic S, Gauthier MG, Mercier JF, Kenward M, McCormick LC, et al: Theory of DNA electrophoresis (approximately 1999-2002(1/2)). Electrophoresis 23:3791-3816, 2002 